\title{
Statistical optimization for passive scalar transport: maximum entropy production versus maximum Kolmogorov-Sinai entropy
}

\author{
M. Mihelich, D. Faranda, B. Dubrulle, and D. Paillard \\ Laboratoire SPHYNX, CEA/IRAMIS/SPEC, CNRS URA 2464, 91191 Gif-sur-Yvette, France \\ Correspondence to: B. Dubrulle (berengere.dubrulle@cea.fr)
}

Received: 18 September 2014 - Published in Nonlin. Processes Geophys. Discuss.: 18 November 2014

Revised: 20 February 2015 - Accepted: 28 February 2015 - Published: 25 March 2015

\begin{abstract}
We derive rigorous results on the link between the principle of maximum entropy production and the principle of maximum Kolmogorov-Sinai entropy for a Markov model of the passive scalar diffusion called the Zero Range Process. We show analytically that both the entropy production and the Kolmogorov-Sinai entropy, seen as functions of a parameter $f$ connected to the jump probability, admit a unique maximum denoted $f_{\max _{\mathrm{EP}}}$ and $f_{\text {max }_{\mathrm{KS}} \text {. The behaviour }}$ of these two maxima is explored as a function of the system disequilibrium and the system resolution $N$. The main result of this paper is that $f_{\max _{\mathrm{EP}}}$ and $f_{\text {max }_{\mathrm{KS}}}$ have the same Taylor expansion at first order in the deviation from equilibrium. We find that $f_{\text {max }_{\mathrm{EP}}}$ hardly depends on $N$ whereas $f_{\text {max }_{\mathrm{KS}}}$ depends strongly on $N$. In particular, for a fixed difference of potential between the reservoirs, $f_{\max }(N)$ tends towards a non-zero value, while $f_{\text {max }_{\mathrm{KS}}}(N)$ tends to 0 when $N$ goes to infinity. For values of $N$ typical of those adopted by Paltridge and climatologists working on maximum entropy production $(N \approx 10-100)$, we show that $f_{\max _{\mathrm{EP}}}$ and $f_{\text {max }_{\mathrm{KS}}}$ coincide even far from equilibrium. Finally, we show that one can find an optimal resolution $N_{*}$ such that $f_{\max _{\mathrm{EP}}}$ and $f_{\max _{\mathrm{KS}}}$ coincide, at least up to a second-order parameter proportional to the non-equilibrium fluxes imposed to the boundaries. We find that the optimal resolution $N^{*}$ depends on the non-equilibrium fluxes, so that deeper convection should be represented on finer grids. This result points to the inadequacy of using a single grid for representing convection in climate and weather models. Moreover, the application of this principle to passive scalar transport parametrization is therefore expected to provide both the value of the optimal flux, and of the optimal number of degrees of freedom (resolution) to describe the system.
\end{abstract}

\section{Introduction}

A major difficulty in the modelling of nonlinear geophysical or astrophysical processes is the taking into account of all the relevant degrees of freedom. For example, fluid motions obeying Navier-Stokes equations usually require of the order of $N=R e^{9 / 4}$ modes to faithfully describe all scales between the injection scale and the dissipative scale (Frisch, 1995). In atmosphere, or ocean, where the Reynolds number exceeds $10^{9}$, this amounts to $N=10^{20}$, a number too large to be handled by any existing computers (Wallace and Hobbs, 2006). The problem is even more vivid in complex systems such as planetary climate, where the coupling of litho- bio- and cryosphere with ocean and atmosphere increases the number of degrees of freedom beyond any practical figure. This justifies the long historical tradition of parametrization and statistical model reduction, to map the exact equations describing the system onto a set of simpler equations involving few degrees of freedom. The price to pay is the introduction of free parameters, describing the action of discarded degrees of freedom, that need to be prescribed.

When the number of free parameters is small, their prescription can be successfully done empirically through calibrating experiments or by a posteriori tuning (Rotstayn, 2000). When the number of parameters is large, such as in climate models where it reaches several hundreds (Murphy et al., 2004), such empirical procedure is inapplicable, because it is impossible to explore the whole parameter space. In that respect, it is of great interest to explore an alternative road to parametrization via application of a statistical optimization principle, such as minimizing or maximizing of a suitable cost functional. As discussed by Turkington (2013) and Pascale et al. (2012), this strategy usually leads to closed reduced equations with adjustable parameters in the closure 
appearing as weights in the cost functional that can be computed explicitly. A renowned example in climate is given by a principle of maximum entropy production (MEP) that allowed (Paltridge, 1975) to derive the distribution of heat and clouds at the Earth surface with reasonable accuracy, without any parameters and with a model of a dozen of degrees of freedom (boxes). Since then, refinements of the Paltridge model have been suggested to increase its generality and range of prediction (Herbert et al., 2011). MEP states that a stationary non-equilibrium system chooses its final state in order to maximize the entropy production as is explained in Martyushev and Seleznev (2006). Rigorous justifications of its application have been searched using e.g. information theory (Dewar and Maritan, 2014) without convincing success. More recently, we have used the analogy of the climate box model of Paltridge with the asymmetric exclusion Markov process (ASEP) to establish numerically a link between the MEP and the principle of maximum Kolmogorov-Sinai entropy (MKS) (Mihelich et al., 2014). The MKS principle is a relatively new concept which extends the classical results of equilibrium physics (Monthus, 2011). This principle applied to Markov chains provides an approximation of the optimal diffusion coefficient in transport phenomena (GómezGardeñes and Latora, 2008) or simulates random walk on irregular lattices (Burda et al., 2009). It is therefore a good candidate for a physically relevant cost functional in passive scalar modelling.

The goal of the present paper is to derive rigorous results on the link between MEP and MKS using a Markov model of the passive scalar diffusion called the Zero Range Process (Andjel, 1982). We find that there exists an optimal resolution $N_{*}$ such that both maxima coincide to second order in the distance from equilibrium. The application of this principle to passive scalar transport parametrization is therefore expected to provide both the value of the optimal flux, and of the optimal number of degrees of freedom (resolution) to describe the system. This suggests that the MEP and MKS principle may be unified when the Kolmogorov-Sinai entropy is defined on opportunely coarse-grained partitions.

\section{From passive scalar equation to ZRP model}

The equation describing the transport of a passive scalar like temperature in a given velocity field $u(x, t)$ reads as

$\partial_{t} T+u \partial_{x} T=\kappa \partial_{x}^{2} T$,

with appropriate boundary conditions, or equivalently, in non-dimensional form:

$\partial_{t} T+u \partial_{x} T=\frac{1}{R e P r} \partial_{x}^{2} T$,

where $\kappa, R e$ and $P r$ are respectively the molecular diffusivity, the Reynolds and the Prandtl number. To solve this equation, one must know both the velocity field and the boundary conditions, and use as many number of modes as necessary to describe the range of scales up to the scales at which molecular diffusivity takes place, i.e. roughly $(\operatorname{RePr})^{3 / 2}$ modes, where $R e$ is the Reynolds number of the convective flow, and $P r$ is its Prandtl number. In geophysical flows, this number is too large to be handled even numerically (Troen and Mahrt, 1986). Moreover, in typical climate studies, the velocity flow is basically unknown as it must obey a complicated equation involving the influence of all the relevant climate components. In order to solve the equation, one must necessarily prescribe the heat flux $f=-u T+\kappa \nabla T$. The idea of Paltridge was then to discretize the passive scalar equation in boxes and prescribe the heat flux $f_{i(i+1)}$ between boxes $i$ and $i+1$ by maximizing the associated thermodynamic entropy production $\dot{S}=\sum_{i} f_{i(i+1)}\left(\frac{1}{T_{i+1}}-\frac{1}{T_{i}}\right)$. Here, we slightly modify the Paltridge discretization approximation to make it amenable to rigorous mathematical results on Markov chains. For simplicity, we stick to a one-dimensional case (corresponding to boxes varying only in latitude) and impose the boundary conditions through two reservoirs located at each end of the chain (mimicking the solar heat flux at pole and equator). We consider a set of $N$ boxes that can contain an arbitrary number $n \in \mathbb{N}$ of particles. We then allow transfer of particles in between two adjacent boxes via decorrelated jumps (to the right or to the left) following a 1-D Markov dynamics governed by a coupling with the two reservoirs imposing a difference of chemical potential at the ends. The resulting process is called the Zero Range Process (ZRP) (Andjel, 1982). The different jumps are described as follows. At each time step a particle can jump right with probability $p w_{n}$ or jump left with probability $q w_{n}$ where $w_{n}$ is a parameter depending of the number of particles inside the box. Physically it represents the interactions between particles. At the edges of the lattice the probability rules are different: at the left edge a particle can enter with probability $\alpha$ and exit with probability $\gamma w_{n}$ whereas at the right edge a particle can exit with probability $\beta w_{n}$ and enter with probability $\delta$. Choices of different $w_{n}$ give radically different behaviours. For example $w_{n}=1+b / n$ where $b \geq 0$ described condensation phenomena (Großkinsky et al., 2003) whereas $w_{1}=w$ and $w_{n}=1$ if $n \geq 2$ has been used to modelled road traffic. We consider in this paper the particular case where $w=1$ by convenience of calculation. Moreover, without loss of generality we will take $p \geq q$ which corresponds to a particle flow from the left to the right and denote $f=p-q$. After a sufficiently long time the system reaches a non-equilibrium steady state. The interest of this toy model is that it is simple enough so that exact computations are analytically tractable.

Taking the continuous limit of this process, it may be checked that the fugacity $z$, which is a quantity related to the average particle density (see Eq. 9 below) of stationary solutions of a system consisting of boxes of size $\frac{1}{N}$, follows 
the continuous equation (Levine et al., 2005) :

$f \frac{\partial z}{\partial x}-\frac{1}{2 N} \frac{\partial^{2} z}{\partial x^{2}}=0$,

corresponding to a stationary solution of a non-dimensional passive scalar equation with non-dimensional velocity $f$ and a non-dimensional diffusivity $\frac{1}{R e P r}=\frac{1}{2 N}$. Therefore, the fugacity of the Zero Range Process is a passive scalar obeying a convection-diffusion equation, with advection velocity controlled by the probability to jump to the right or to the left, and diffusivity controlled by the number of boxes: the larger the number of boxes (the finer the resolution) the smaller the diffusivity. This observation illuminates the well-known observation that the numerical diffusion of a discrete model of diffusion is inversely proportional to the resolution. The parameter $f$ controls the regime: $f=0$ corresponds to a purely conductive regime whereas the larger $f$ the more convective the regime. In the sequel, we calculate the entropy production and the Kolmogorov-Sinai entropy function of $f$. These two quantities reach a maximum denoted respectively $f_{\text {max }_{\mathrm{EP}}}$ and

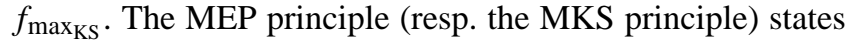
that the system will choose $f=f_{\text {max }_{\mathrm{EP}}}\left(\operatorname{resp} f=f_{\text {max }_{\mathrm{KS}}}\right)$.

We will first show that numerically $f_{\text {max }_{\mathrm{EP}}} \approx f_{\text {max }_{\mathrm{KS}}}$ even far from equilibrium for a number of boxes $N$ roughly corresponding to the resolution taken by Paltridge (1975) in his climate model. This result is similar to what we found for the ASEP model (Mihelich et al., 2014) and thus gives another example of a system in which the two principles are equivalent. Moreover we will see analytically that $f_{\max _{\text {EP }}}$ and $f_{\text {max }_{K S}}$ have the same behaviour in first order in the difference of the chemical potentials between the two reservoirs for $N$ large enough. These results provide a better understanding of the relationship between the MEP and the MKS principles.

\section{Notation and useful preliminary results}

This Markovian process is a stochastic process with an infinite number of states in bijection with $\mathbb{N}^{N}$. In fact, each state can be written $n=\left(n_{1}, n_{2}, \ldots, n_{N}\right)$ where $n_{i}$ is the number of particle lying in site $i$. We call $P_{n}$ the stationary probability to be in state $n$. In order to calculate this probability it is easier to use a quantum formalism than the Markovian formalism (Domb, 2000; Levine et al., 2005).

The probability to find $m$ particles in the site $k$ is equal to $p_{k}\left(n_{k}=m\right)=\frac{z_{k}^{m}}{Z_{k}}$ where $Z_{k}$ is the analogue of the grand canonical repartition function and $z_{k}$ is the fugacity between 0 and 1 . Moreover $Z_{k}=\sum_{i=0}^{\infty} z_{k}^{i}=\frac{1}{1-z_{k}}$. So, finally

$p_{k}\left(n_{k}=m\right)=\left(1-z_{k}\right) z_{k}^{m}$.

We can show that the probability $P$ over the states is the tensorial product of the probability $p_{k}$ over the boxes:

$P=p_{1} \otimes p_{2} \otimes \cdots \otimes p_{N}$.
Thus events $\left(n_{k}=m\right)$ and $\left(n_{k}^{\prime}=m^{\prime}\right)$ for $k \neq k^{\prime}$ are independent and so

$P\left(m_{1}, m_{2}, \ldots, m_{N}\right)=p_{1}\left(n_{1}=m_{1}\right) * \cdots * p_{N}\left(n_{N}=m_{N}\right)$.

So finally

$P\left(m_{1}, m_{2}, \ldots, m_{N}\right)=\prod_{k=1}^{N}\left(1-z_{k}\right) z_{k}^{m_{k}}$.

Moreover, with the Hamiltonian equation found from the quantum formalism we can find the exact values of $z_{k}$ function of the system parameters:

$z_{k}=\frac{\left(\frac{p}{q}\right)^{k-1}[(\alpha+\delta)(p-q)-\alpha \beta+\gamma \delta]-\gamma \delta+\alpha \beta\left(\frac{p}{q}\right)^{N-1}}{\gamma(p-q-\beta)+\beta(p-q+\gamma)\left(\frac{p}{q}\right)^{N-1}}$,

and the flux of particles $c$ :

$c=(p-q) \frac{-\gamma \delta+\alpha \beta\left(\frac{p}{q}\right)^{N-1}}{\gamma(p-q-\beta)+\beta(p-q+\gamma)\left(\frac{p}{q}\right)^{N-1}}$.

Finally, the stationary density is related to the fugacity by the relation

$\rho_{k}=z_{k} \frac{\partial \log Z_{k}}{\partial z_{k}}=\frac{z_{k}}{1-z_{k}}$.

\subsection{Entropy production}

For a system subject to internal forces $X_{i}$ and associated fluxes $J_{i}$ the macroscopic entropy production is well known (Onsager, 1931) and takes the form

$\sigma=\sum_{i} J_{i} \cdot X_{i}$.

The physical meaning of this quantity is a measure of irreversibility: the larger $\sigma$ the more irreversible the system.

In the case of the ZRP, irreversibility is created by the fact that $p \neq q$. We will parametrize this irreversibility by the parameter $f=p-q$ and we will take $p+q=1$. In the remainder of the paper, we take, without loss of generality, $p \leq q$ which corresponds to a flow from left to right. Moreover, the only flux to be considered here is the flux of particles $c$ and the associated force is due to the gradient of the density of particles $\rho: X=\nabla \log \rho$ (Balian, 1992).

Thus, when the stationary state is reached, i.e. when $c$ is constant,

$\sigma=\sum_{i=1}^{N-1} c \cdot\left(\log \left(\rho_{i}\right)-\log \left(\rho_{i+1}\right)\right)=c \cdot\left(\log \left(\rho_{1}\right)-\log \left(\rho_{N}\right)\right)$.

Thus, according to Eqs. (7), (8), (9) and (10) when $N$ tends to $+\infty$ we obtain:

$$
\begin{aligned}
\sigma(f) & =\frac{\alpha f}{f+\gamma}\left(\log \left(\frac{\alpha}{f+\gamma-\alpha}\right)\right. \\
& \left.-\log \left(\frac{(\alpha+\delta) f+\gamma \delta}{f(\beta-\alpha-\delta)+\beta \gamma-\gamma \delta}\right)\right) .
\end{aligned}
$$

Because $f \geq 0$ the entropy production is positive if and only if $\rho_{1} \geq \rho_{N}$ and iff $z_{1} \geq z_{N}$. This is physically coherent be- 
cause fluxes are in the opposite direction of the gradient. We remark that if $f=0$ then $\sigma(f)=0$. Moreover, when $f$ increases $\rho_{1}(f)$ decreases and $\rho_{2}(f)$ increases until they take the same value. Thus there exists $f$, large enough, for which $\sigma(f)=0$. Between these two values of $f$ the entropy production has at least one maximum.

\subsection{Kolmogorov-Sinai entropy}

There are several ways to introduce the Kolmogorov-Sinai entropy which is a mathematical quantity introduced by Kolmogorov and developed by the renowned mathematicians Sinai and Billingsley (Billingsley, 1965). Nevertheless, for a Markov process we can give it a simple physical interpretation: the Kolmogorov-Sinai entropy is the time derivative of the Jaynes entropy (entropy over the path):

$S_{\text {Jaynes }}(t)=-\sum_{\Gamma_{[0, t]}} p_{\Gamma_{[0, t]}} \log \left(p_{\Gamma_{[0, t]}}\right)$,

For a Markov Chain we thus have

$$
S_{\text {Jaynes }}(t)-S_{\text {Jaynes }}(t-1)=-\sum_{(i, j)} \mu_{i_{\text {stat }}} p_{i j} \log \left(p_{i j}\right),
$$

where $\mu_{\text {stat }}=\mu_{i_{\text {stat }}} i=1, \ldots, N$ is the stationary measure and where the $p_{i j}$ are the transition probabilities.

Thus the Kolmogorov-Sinai entropy takes the following form:

$h_{\mathrm{KS}}=-\sum_{(i, j)} \mu_{i_{\mathrm{stat}}} p_{i j} \log \left(p_{i j}\right)$.

For the ZRP, we show in the Appendix that it can be written as

$$
\begin{aligned}
h_{\mathrm{KS}} & =-(\alpha \log \alpha+\delta \log \delta+\gamma \log \gamma+\beta \log \beta \\
& +(N-1)(p \log (p)+q \log (q)))+(p \log (p)+q \log (q)) \\
& \sum_{i=1}^{N}\left(1-z_{i}\right)+(\gamma \log (\gamma)+p \log (p))\left(1-z_{1}\right) \\
& +(\beta \log (\beta)+q \log (q))\left(1-z_{N}\right) .
\end{aligned}
$$

\section{Results}

We start by pointing to some interesting properties of $f_{\text {max }}$ and $f_{\text {max }_{K S}}$, then present numerical experiments on the ZRP model and conclude with some analytical computations.

Note that for $N, \alpha, \beta, \gamma$ and $\delta$ fixed the entropy production as well as the Kolmogorov-Sinai entropy seen as functions of $f$ both admit a unique maximum. When $N$ tends to infinity and $f=0$, using Eq. (7) (i.e. the symmetric case), we find that $z_{1}=\frac{\alpha}{\gamma}$ and $z_{N}=\frac{\delta}{\beta}$. Thus, the system is coupled with two reservoirs with respective chemical potential $\frac{\alpha}{\gamma}$ (left) and $\frac{\delta}{\beta}$ (right). For $\frac{\alpha}{\gamma} \neq \frac{\delta}{\beta}$ the system is out of equilibrium. We assume, without loss of generality, $z_{1} \geq z_{N}$, which corresponds to a flow from left to right. As a measure of deviation from equilibrium we take $s=z_{1}-z_{N}$ : the larger $s$, the more density fluxes we expect into the system.

First we remark that $f_{\text {max }_{\mathrm{EP}}}$ hardly depends on $N$ whereas $f_{\text {max }}$ depends strongly on $N$. This is easily understood because $\sigma$ depends only on $z_{1}$ and $z_{N}$ whereas $h_{\mathrm{KS}}$ depends on all the $z_{i}$. Moreover, the profile of the $z_{i}$ depends strongly on $N$. In particular, for a fixed difference of potential between the reservoirs, $f_{\max }(N)$ tends towards a non-zero value, while $f_{\text {max }_{\mathrm{KS}}}(N)$ tends to 0 when $N$ goes to infinity.

Moreover, $f_{\max _{\mathrm{EP}}}$ and $f_{\text {max }_{\mathrm{KS}}}$ coincide even far from equilibrium for $N$ corresponding to the choice of Paltridge (1975) $N \approx 10 \sim 100$. For $N$ fixed, as large as one wants, and for all $\epsilon$, as small as one wants, there exists $v$ such that for all $s \in[0 ; v]\left|f_{\text {max }_{E P}}-f_{\text {max }_{K S}}\right| \leq \epsilon$.

These observations are confirmed by the results presented in Figs. 1 and 3 where EP and KS are calculated using Eqs. (7) and (15) for $s=0.13$ and three different partitions: $N=20, N=100$ and $N=1000$. The figure shows that $f_{\max }$ and $f_{\text {max }}$ coincide with good approximation for $N=20$ and $N=100$. But then when $N$ increases $f_{\text {max }_{K S}}(N)$ tends to 0 whereas $f_{\max _{E P}}(N)$ tends to a non-zero value.

In Fig. 2 we represent the entropy production (top) and KS entropy (bottom) as functions of $f$ for $N=1000$ and for three value of $s: s=0.13 ; s=0.2 ; s=0.04$. This supports the claim that for $N$ fixed, we can try different values of $s$ such that $s \in[0 ; \nu]\left|f_{\max _{E P}}-f_{\max _{K S}}\right| \leq \epsilon$. Figure 3 shows that $\Delta f_{\max }$ is minimum when the system is close to equilibrium whereas the further the system is from equilibrium (when $s$ increases) the more $\Delta f_{\max }$ increases. Moreover, the optimal resolution where $f_{\text {max }_{E P}}$ and $f_{\text {max }_{K S}}$ coincide is approximately 10-100. Then $\Delta f_{\max }$ is maximum at $N=500$ and $s=0.05 . \Delta f_{\max }$ is obviously linear in $s$, for small values of $s$, but the behaviour with $N$ is more complicated.

Such numerical investigations help to understand why $f_{\max _{\mathrm{KS}}}(N)$ and $f_{\text {max }_{\mathrm{EP}}}(N)$ have different behaviour functions of $N$, and why for $N$ large enough $f_{\text {max }_{\mathrm{KS}}}$ and $f_{\text {max }_{\mathrm{EP}}}$ have the same behaviour of first order in the deviation from equilibrium measured by the parameter $s$. We will see that we can get a precise answer to such questions by doing calculations and introducing a sort of hydrodynamics approximation.

\subsection{Taylor expansion}

From Eq. (15) it is apparent that $f_{\text {max }_{K S}}$ depends on $N$ whereas from Eq. (10) we get that $f_{\text {max }_{E P}}$ hardly depends on $N$. Indeed there is a difference between $f_{\text {max }_{\mathrm{EP}}}$ and $f_{\text {max }_{K S}}$, i.e. a difference between the two principles for the ZRP. Nevertheless, we have seen numerically that there is a range of $N$, namely $N \approx 10-100$ for which the maxima fairly coincide.

Using Eqs. (15), (7), and (11) we compute analytically the Taylor expansion of $f_{\max _{\mathrm{EP}}}$ and $f_{\text {max }_{\mathrm{KS}}}$ in $s$. We will show the main result: $f_{\text {max }_{E P}}$ and $f_{\text {max }_{K S}}$ have the same Taylor expansion in first order in $s$ for $N$ large enough. Their Taylor 

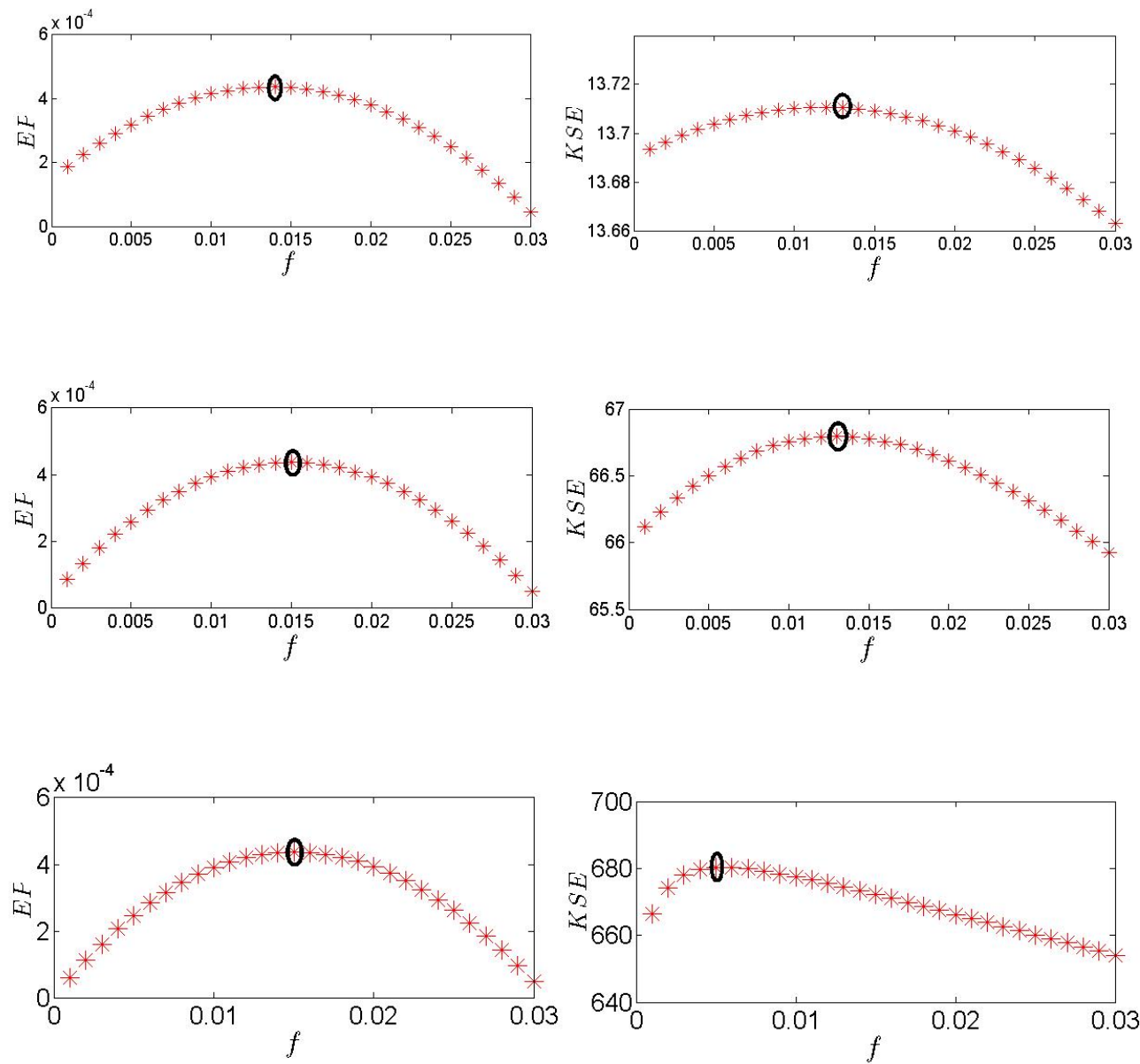

Figure 1. Entropy production calculated using Eq. (11) (left) and KS entropy calculated using Eqs. (7) and (15) (right) as functions of $f$ for $s=0.13$ and respectively $N=20, N=100$ and $N=1000$.

expansions are different up to the second order in $s$ but there exists an $N$, i.e. a resolution, such that $f_{\text {max }_{\mathrm{EP}}}$ and $f_{\text {max }_{\mathrm{KS}}}$ coincident up to the second order.

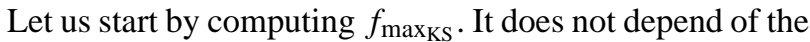
constant terms of $h_{\mathrm{KS}}$ in Eq. (15) and therefore we need only concern ourselves with

$$
\begin{aligned}
& -(p \log (p)+q \log (q))\left(\sum_{i=1}^{N}\left(z_{i}\right)-1\right)+(\gamma \log (\gamma) \\
& +p \log (p))\left(1-z_{1}\right)+(\beta \log (\beta)+q \log (q))\left(1-z_{N}\right) \\
& =N \cdot H(f, N, \alpha, \gamma, \beta, \delta) .
\end{aligned}
$$

Using Eq. (7), the expression of $H(f, N, \alpha, \gamma, \beta, \delta)$ takes an easy form. To simplify the calculations, we restrict the space of parameter by assuming $\alpha+\gamma=1$ and $\beta+\delta=1$ and we parametrize the deviation from equilibrium by the parameter $\bar{s}=\alpha-\delta$. Moreover let us denote $a=\frac{1}{N}$. Thus, we have $H(f, N, \alpha, \gamma, \beta, \delta)=H(f, a, \alpha, \bar{s})$. In order to know the Taylor expansion to the first order in $\bar{s}$ of $f_{\text {max }_{K S}}$ we develop $H(f, a, \alpha, \bar{s})$ up to the second order in $f$; i.e. we have $H(f, a, \alpha, \bar{s})=C+B f+A f^{2}+o\left(f^{2}\right)$. Then we find $f_{\max _{\mathrm{KS}}}=-B / 2 A$ which we will develop in the power of $\bar{s}$. This is consistent if we assume $f \ll a$.

After some tedious but straightforward calculations, we get at the first order in $\bar{s}$ :

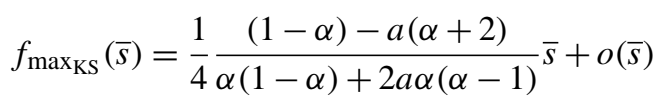

and so

$$
f_{\max _{\mathrm{KS}}}(\bar{s})=\frac{1}{4 \alpha} \bar{s}+\frac{3 a}{4(\alpha-1)} \bar{s}+o(\bar{s})+o(a \bar{s}) .
$$

We repeat the same procedure starting from Eq. (11) and we obtain

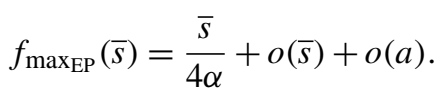

Thus, since $a=\frac{1}{N} \ll 1$ the behaviour of $f_{\max _{K S}}(\bar{s})$ and

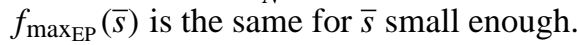

We remark that we can strictly find the same result by solving the hydrodynamics continuous approximation given by Eq. (3). This equation is a classical convection-diffusion 

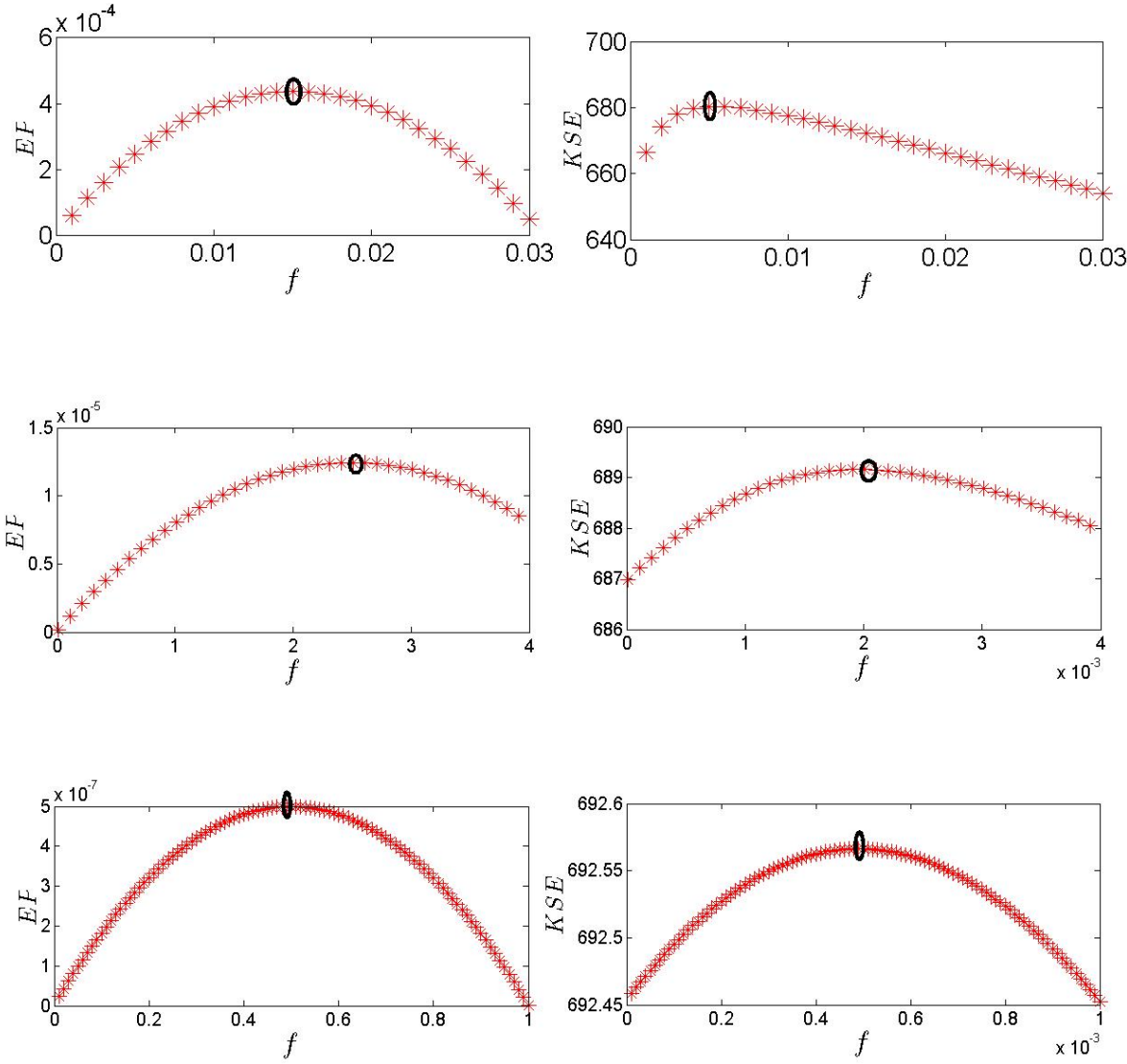

Figure 2. Entropy production (left) and KS entropy (right) as functions of $f$ for $N=1000$ and respectively $s=0.13, s=0.2$ and $s=0.04$.

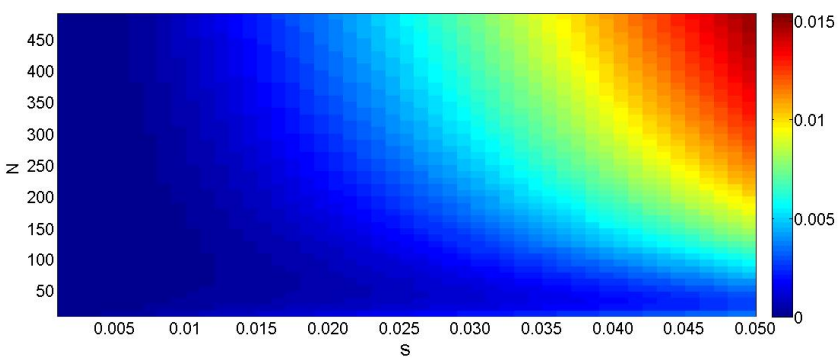

Figure 3. 2-D plot representing $\Delta f_{\max }=f_{\max _{\mathrm{EP}}}-f_{\max _{\mathrm{KS}}}$ in the $(N, s)$ space.

equation. We remark that, by varying $f$, we change the convective behaviour: $f=0$ corresponds to a purely diffusive regime whereas by increasing $f$ we enhance the role of convection. If the system is near equilibrium then $f_{\max _{\mathrm{EP}}} \approx$ $f_{\text {max }_{\mathrm{KS}}} \approx 0$ and the system is purely diffusive. When the system is out of equilibrium $f_{\text {max }_{E P}}$ and $f_{\text {max }_{K S}}$ are different from 0 and the system corresponds to an (optimal) trade-off between purely diffusive and convective behaviour.

One can verify this numerically: we first calculate the exact values of the entropy production function of $f$ using Eq. (7) and the Kolmogorov-Sinai entropy function of $f$ us- ing Eqs. (7) and (15). Then we approximate these two curves with a cubic spline approximation in order to find $f_{\text {max }_{\mathrm{EP}}}$ and $f_{\text {max }_{\mathrm{KS}} \text {. }}$

In order to find the optimal resolution $N_{*}$ we can go one step further by expanding $f_{\max _{E P}}$ and $f_{\max _{K S}}$ up to the second order in $\bar{s}$ :

$$
\begin{aligned}
& f_{\max _{\mathrm{EP}}}(\bar{s})=\frac{\bar{s}}{4 \alpha}+\frac{\bar{s}^{2}(\alpha+1)}{8 \alpha^{2}(\alpha-1)}+o\left(\overline{s^{2}}\right)+o(a), \\
& f_{\max _{\mathrm{KS}}}(\bar{s})=\frac{1}{4} \frac{(1-\alpha)-a(\alpha+2)}{\alpha(1-\alpha)+2 a \alpha(\alpha-1)} \bar{s} \\
& +\frac{(1-\alpha)^{2}+a\left(\alpha^{2}-2 \alpha+1\right)}{8 \alpha^{2}(\alpha-1)^{2}(1-2 a)} \bar{s}^{2}+o\left(\bar{s}^{2}\right) .
\end{aligned}
$$

Thus, $f_{\text {max }_{\mathrm{EP}}}$ and $f_{\text {max }_{\mathrm{KS}}}$ coincide in second order in $\bar{s}$ iff $a$ satisfies the quadratic equation

$$
\begin{aligned}
& \left(4 \alpha-6 \alpha^{2}+6 \alpha^{3}-4 \bar{s}+3 \alpha^{2} \bar{s}\right) a^{2} \\
& -\frac{1}{2}\left(8 \alpha-8 \bar{s}+3 \alpha^{2} \bar{s}-6 \alpha^{2}+6 \alpha^{3}\right) a-(1-\alpha)=0 .
\end{aligned}
$$



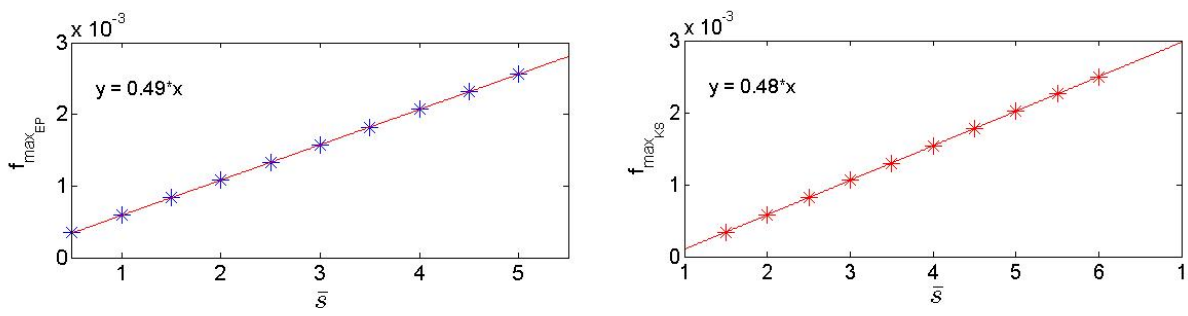

Figure 4. $f_{\max _{\mathrm{EP}}}$ (left) and $f_{\max _{\mathrm{KS}}}$ (right) as functions of $\bar{s}$ for $\alpha=0.5$ and $N=100$. Both $f_{\max _{\mathrm{KS}}}$ and $f_{\max _{\mathrm{EP}}}$ have a linear behaviour with slope respectively 0.48 and 0.49 , close to $\frac{1}{4 \alpha}=0.5$.
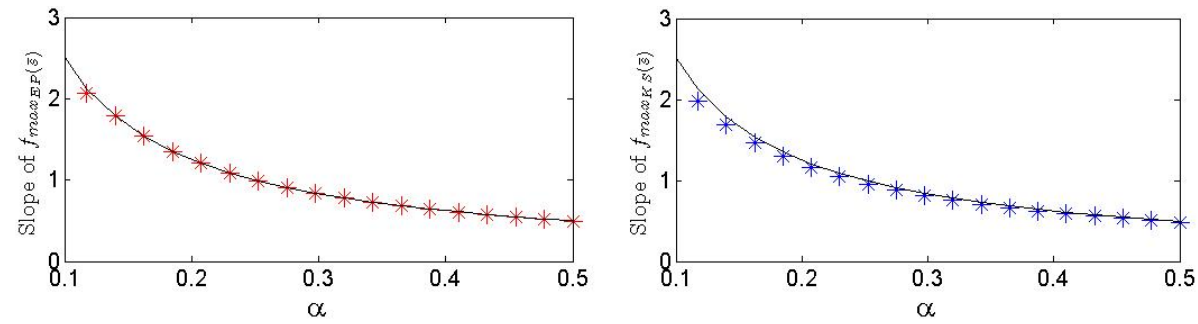

Figure 5. $f_{\max _{\mathrm{KS}}(\bar{s})}($ left $)$ and $f_{\max _{\mathrm{EP}}(\bar{s})}\left(\right.$ right) as functions of $\alpha$ and in black the curve $f(\bar{s})=\frac{1}{4 \alpha} \bar{s}$. Note that the approximation $f_{\text {max }}(\bar{s}) \approx$ $f_{\max _{\mathrm{EP}}}(\bar{s}) \approx \frac{1}{4 \alpha} \bar{s}$ is good.

This equation has a unique positive solution because the leading coefficient is positive for $s$ small enough $(4 \alpha-$ $\left.6 \alpha^{2}+6 \alpha^{3}-4 \bar{s}+3 \alpha^{2} \bar{s}\right) \geq 0$ and the constant term is negative $-(1-\alpha) \leq 0$. We remark that the optimal resolution $N_{*}=\frac{1}{a *}$ depends on the parameters of the system namely on the degree of non-equilibrium. This fact can be the explanation for two well-known issues in climate/weather modelling. First, it explains that, when downgrading or upgrading the resolution of convection models, the relevant parameters must be changed as they depend on the grid size. Second, it suggests that if the resolution is well tuned to represent a particular range of convective phenomena, it might fail in capturing the dynamics out of this range: since finer grids are needed to better represent deep convection phenomena, the deviations between model and observations observed in the distribution of extreme convective precipitation may be due to an inadequacy of the grid used.

\section{Conclusions}

We have shown how a simple 1-D Markov process, the Zero Range Process, can be used to obtain rigorous results on the problem of parametrization of the passive scalar transport problem, relevant to many geophysical applications including temperature distribution in climate modelling. Using this model, we have derived rigorous results on the link between a principle of maximum entropy production and the principle of maximum Kolmogorov-Sinai entropy using a Markov model of the passive scalar diffusion called the Zero Range Process. The Kolmogorov-Sinai entropy seen as a function of the convective velocity admits a unique maximum. We show analytically that both have the same Taylor expansion at the first order in the deviation from equilibrium. The behaviour of these two maxima is explored as a function of the resolution $N$ (equivalent to the number of boxes, in the box approximation). We found that, for a fixed difference of potential between the reservoirs, the maximal convective velocity predicted by the maximum entropy production principle tends towards a non-zero value, while the maximum predicted using Kolmogorov-Sinai entropy tends to 0 when $N$ goes to infinity. For values of $N$ typical of those adopted by climatologists $(N \approx 10-100)$, we show that the two maxima nevertheless coincide even far from equilibrium. Finally, we show that there is an optimal resolution $N_{*}$ such that the two maxima coincide to second order in $\bar{s}$, a parameter proportional to the non-equilibrium fluxes imposed to the boundaries. The fact that the optimal resolution depends on the intensity of the convective phenomena to be represented, points to new interesting research avenues, e.g. the introduction of convective models with adaptive grids optimized with maximum entropy principles on the basis of the convective phenomena to be represented.

The application of this principle to passive scalar transport parametrization is therefore expected to provide both the value of the optimal flux, and of the optimal number of degrees of freedom (resolution) to describe the system. It would be interesting to apply it to a more realistic passive scalar transport problem, to see if it would yield a model that could be numerically handled (i.e. corresponding to a number of bows that is small enough to be handled by present computers). In view of applications to atmospheric convection, it 
would be interesting to apply this procedure to the case of an active scalar, coupled with a Navier-Stokes equation for the velocity. In such a case, the role of $f$ will be played by the turbulent subgrid Reynolds stresses. The heat fluxes and $N^{*}$ will be fixed by the coarse-graining length, and the optimization procedure will in principle provide the optimum subgrid Reynolds stresses at a given resolution $N$. Moreover, by imposing coincidence of MKS and MEP, one could get both the Reynolds stresses, heat fluxes and the optimum resolution. Further, on the theoretical side, it will be interesting to study whether for general dynamical systems, there exists a smart way to coarse grain the Kolmogorov-Sinai entropy such that its properties coincide with the thermodynamic entropy production. This would eventually justify the use of the MEP principle and explain the deviations as well as the different representations of it due to the dependence of the dynamic (Kolmogorov-Smirnov-Tsallis-Jaynes) entropies on the kind of partition adopted. 


\section{Appendix A: Computation of the K-S entropy}

In this appendix, we compute the Kolmogorov-Sinai entropy for the Zero Range Process, starting from its definition in Eq. (14). In the frame of our Zero Range Process, we use Eqs. (14) and (6) to write it as

$$
\begin{aligned}
& h_{\mathrm{KS}}=-\sum_{i} \mu_{i_{\mathrm{stat}}} \sum_{j} p_{i j} \log \left(p_{i j}\right)=-\sum_{m_{1}=0}^{+\infty} \ldots \sum_{m_{N}=0}^{+\infty} \\
& P\left(m_{1}, m_{2}, \ldots, m_{N}\right) \sum_{j} p_{\left(m_{1}, \ldots, m_{N}\right) \rightarrow j} \log \left(p_{\left(m_{1}, \ldots, m_{N}\right) \rightarrow j}\right) \\
& =-\sum_{m_{1}=0}^{+\infty} P\left(m_{1}\right) \ldots \sum_{m_{N}=0}^{+\infty} \\
& P\left(m_{N}\right) \sum_{j} p_{\left(m_{1}, \ldots, m_{N}\right) \rightarrow j} \log \left(p_{\left(m_{1}, \ldots, m_{N}\right) \rightarrow j}\right)
\end{aligned}
$$

We thus have to calculate $\sum_{j} \quad p_{\left(m_{1}, \ldots, m_{N}\right) \rightarrow j}$ $\log \left(p_{\left.\left(m_{1}, \ldots, m_{N}\right) \rightarrow j\right)}\right)$ that we will refer to as $(*)$. We will take $p+q=\alpha+\delta=\beta+\gamma=1$ and $\mathrm{d} t=\frac{1}{N}$ in order to neglect the probabilities to stay in the same state compare to the probabilities of changing state. There are five different cases to consider:

1. if $\forall i m_{i} \geq 1$ so the possible transitions are:

$\left(m_{1}, m_{2}, \ldots, m_{N}\right) \rightarrow\left(m_{1} \pm 1, m_{2}, \ldots, m_{N}\right)$ with respective probabilities $\alpha$ and $\delta$;

$\left(m_{1}, m_{2}, \ldots, m_{N}\right) \rightarrow\left(m_{1}, m_{2}, \ldots, m_{N} \pm 1\right)$ with respective probabilities $\gamma$ and $\beta$;

and $\left(m_{1}, \ldots, m_{k}, \ldots, m_{N}\right) \rightarrow\left(m_{1}, \ldots, m_{k} \pm 1, \ldots, m_{N}\right)$ with respective probabilities $p$ and $q$;

thus,

$$
\begin{aligned}
(*) & =\alpha \log \alpha+\delta \log \delta+\gamma \log \gamma+\beta \log \beta \\
& +(N-1)(p \log (p)+q \log (q)) ;
\end{aligned}
$$

2. if $m_{1} \geq 1$ and $m_{N} \geq 1$ and let $i$ be the number of $m_{i}$ between 2 and $N-1$ equal to 0 . With the same argument as previously we have

$$
\begin{aligned}
(*) & =\alpha \log \alpha+\delta \log \delta+\gamma \log \gamma+\beta \log \beta \\
& +(N-1-i)(p \log (p)+q \log (q)) ;
\end{aligned}
$$

3. if $m_{1}=0$ and $m_{N} \geq 1$ and let $i$ the number of $m_{i}$ between 2 and $N-1$ equal to 0 we have

$$
\begin{aligned}
(*) & =\alpha \log \alpha+\delta \log \delta+\beta \log \beta \\
& +(N-2-i) p \log (p)+(N-1-i) q \log (q) ;
\end{aligned}
$$

4. the same applies if $m_{1} \geq 1$ and $m_{N}=0$ and let $i$ the number of $m_{i}$ between 2 and $N-1$ equal to 0 we have

$$
\begin{aligned}
(*) & =\alpha \log \alpha+\delta \log \delta+\gamma \log \gamma \\
& +(N-1-i) p \log (p)+(N-2-i) q \log (q) ;
\end{aligned}
$$

5. finally, if $m_{1}=0$ and $m_{N}=0$ and let $i$ the number of $m_{i}$ between 2 and $N-1$ equal to 0 we have

$$
\begin{aligned}
(*) & =\alpha \log \alpha+\delta \log \delta \\
& +(N-2-i)(p \log (p)+q \log (q) .
\end{aligned}
$$

Using Eq. (4) we find that $P\left(m_{k}=0\right)=1-z_{k}$ and $\sum_{i=1}^{+\infty} P\left(m_{k}=i\right)=z_{k}$.

We thus obtain that

$$
\begin{aligned}
h_{\mathrm{KS}} & =-(\alpha \log \alpha+\delta \log \delta+\gamma \log \gamma+\beta \log \beta) \\
& +(N-1)(p \log (p)+q \log (q)) \\
& +(p \log (p)+q \log (q)) \\
& \left(\sum_{r=0}^{N} r \sum_{i_{1} \ldots i_{N} i=i_{1}, \ldots i_{r}} \prod_{i \neq i_{1} \ldots i_{r}}\left(1-z_{i}\right) \prod_{i} z_{i}\right) \\
& +(\gamma \log (\gamma)+p \log (p)) z_{N}\left(1-z_{1}\right) \\
& \left(\sum_{i_{2} \ldots i_{N-1} i=i_{2}, \ldots i_{r}}\left(1-z_{i}\right) \prod_{i \neq i_{2} \ldots i_{r}} z_{i}\right) \\
& +(\beta \log (\beta)+q \log q) z_{1}\left(1-z_{N}\right) \\
& \left(\sum_{i_{2} \ldots i_{N-1} i=i_{2}, \ldots i_{r}}\left(1-z_{i}\right) \prod_{i \neq i_{2} \ldots i_{r}} z_{i}\right) \\
& +(\beta \log (\beta)+\gamma \log \gamma+p \log p+q \log q) \\
& \left(\sum_{i_{2} \ldots i_{N-1} i=i_{2}, \ldots i_{r}}\left(1-z_{i}\right) \prod_{i \neq i_{2} \ldots i_{r}} z_{i}\right) .
\end{aligned}
$$

This expression, though complicated at first sight, can be simplified. Indeed, interested in the function $F(a)=$ $\prod_{1}^{N}\left(z_{k}+a\left(1-z_{k}\right)\right)$ and by deriving subject to $a$ we show that

$\sum_{r=0}^{N} r \sum_{i_{1} \ldots i_{N}} \prod_{i=i_{1}, \ldots i_{r}}\left(1-z_{i}\right) \prod_{i \neq i_{1} \ldots i_{r}} z_{i}=\sum_{i=1}^{N}\left(1-z_{i}\right)$.

Thus we can simplify the earlier equation to obtain

$$
\begin{aligned}
h_{\mathrm{KS}} & =-(\alpha \log \alpha+\delta \log \delta+\gamma \log \gamma+\beta \log \beta \\
& +(N-1)(p \log (p)+q \log (q))) \\
& +(p \log (p)+q \log (q)) \sum_{i=1}^{N}\left(1-z_{i}\right) \\
& +(\gamma \log (\gamma)+p \log (p))\left(1-z_{1}\right) \\
& +(\beta \log (\beta)+q \log (q))\left(1-z_{N}\right) .
\end{aligned}
$$


Acknowledgements. M. Mihelich thanks IDEEX Paris Saclay for financial support.

Edited by: J. M. Redondo

Reviewed by: two anonymous referees

\section{References}

Andjel, E. D.: Invariant measures for the zero range process, Ann. Probabil., 525-547, 1982.

Balian, R.: Physique statistique et themodynamique hors équilibre, Ecole Polytechnique, 1992.

Billingsley, P.: Ergodic theory and information, Wiley, 1965.

Burda, Z., Duda, J., Luck, J. M., and Waclaw, B.: Localization of the maximal entropy random walk, Phys. Rev. Lett., 102, 160602, 2009.

Dewar, R. C. and Maritan, A.: A theoretical basis for maximum entropy production, in: Beyond the Second Law, 49-71, Springer, 2014.

Domb, C.: Phase transitions and critical phenomena, Vol. 19, Academic Press, 2000

Frisch, U.: Turbulence: the legacy of AN Kolmogorov, Cambridge University Press, 1995.

Gómez-Gardeñes, J. and Latora, V.: Entropy rate of diffusion processes on complex networks, Phys. Rev. E, 78, 065102, 2008.

Großkinsky, S., Schütz, G. M., and Spohn, H.: Condensation in the zero range process: stationary and dynamical properties, J. Stat. Phys., 113, 389-410, 2003.

Herbert, C., Paillard, D., Kageyama, M., and Dubrulle, B.: Present and Last Glacial Maximum climates as states of maximum entropy production, Q. J. Roy. Meteorol. Soc., 137, 1059-1069, 2011.

Levine, E., Mukamel, D., and Schütz, G.: Zero-range process with open boundaries, J. Stat. Phys., 120, 759-778, 2005.
Martyushev, L. M. and Seleznev, V. D.: Maximum entropy production principle in physics, chemistry and biology, Phys. Rep., 426, 1-45, 2006.

Mihelich, M., Dubrulle, B., Paillard, D., and Herbert, C.: Maximum Entropy Production vs. Kolmogorov-Sinai Entropy in a Constrained ASEP Model, Entropy, 16, 1037-1046, 2014.

Monthus, C.: Non-equilibrium steady states: maximization of the Shannon entropy associated with the distribution of dynamical trajectories in the presence of constraints, J. Stat. Mech., p. P03008, 2011.

Murphy, J. M., Sexton, D. M., Barnett, D. N., Jones, G. S., Webb, M. J., Collins, M., and Stainforth, D. A.: Quantification of modelling uncertainties in a large ensemble of climate change simulations, Nature, 430, 768-772, 2004.

Onsager, L.: Reciprocal relations in irreversible processes. I., Phys. Rev., 37, 2265-2279, 1931.

Paltridge, G. W.: Global dynamics and climate-a system of minimum entropy exchange, Q. J. Roy. Meteorol. Soc., 101, 475-484, 1975.

Pascale, S., Gregory, J. M., Ambaum, M. H., and Tailleux, R.: A parametric sensitivity study of entropy production and kinetic energy dissipation using the FAMOUS AOGCM, Clim. Dynam., 38, 1211-1227, 2012.

Rotstayn, L. D.: On the "tuning" of autoconversion parameterizations in climate models, J. Geophys. Res.-Atmso. (1984-2012), 105, 15495-15507, 2000.

Troen, I. and Mahrt, L.: A simple model of the atmospheric boundary layer; sensitivity to surface evaporation, Boundary-Lay. Meteorol., 37, 129-148, 1986.

Turkington, B.: An optimization principle for deriving nonequilibrium statistical models of hamiltonian dynamics, J. Stat. Phys. 152, 569-597, 2013.

Wallace, J. M. and Hobbs, P. V.: Atmospheric science: an introductory survey, Vol. 92, Academic press, 2006. 